\title{
A SPECTRAL SEQUENCE FOR GROUP PRESENTATIONS WITH APPLICATIONS TO LINKS
}

BY

\author{
SELMA WANNA ${ }^{1}$
}

\begin{abstract}
A spectral sequence is associated with any presentation of a group $G$. It turns out that this spectral sequence is independent of the chosen presentation. In particular if $G$ is the fundamental group of a link $L$ in $R^{3}$ the spectral sequence leads to invariants that compare to the Milnor invariants of $L$.
\end{abstract}

0. Introduction. Recently Stallings used the cobar construction of a resolution to associate to each group $G$ a 2 nd quadrant spectral sequence $E_{-s, t}^{r}$ which is 0 for $s>t$ and which satisfies $E_{-s, s}^{\infty}=I^{s} G / I^{s+1} G$ where $I G$ is the fundamental ideal of $G$ [9]. Here we present a different construction with all the properties mentioned above but with some advantages. First, it can be read off from any group presentation. Second, $E_{-s, t}^{r}=0$ for $t \geqslant s+2$. In Stallings' sequence one has no information on those terms (and they are definitely not zero). Third, and most important, the $E_{-s, s}^{r}$ and $E_{-s, s+1}^{r}$ terms are related to the Baer invariants of $G$ [1]. This is better than the results in [5] which do depend upon the presentation while ours do not.

We describe our sequence in $\$ 1$; in $\S 2$ we show that the sequence is intrinsically defined by using the results of [4]. In $\$ 3$ we apply our results to the theory of links in $R^{3}$.

[The referee would like to thank J. Ratcliffe for pointing out to him the existence of [4]. Thanks to it, the referee was able to improve some results and prove a conjecture of the author's (the main theorem).]

1. The spectral sequence of a presentation.

(1.0) We shall consider complexes of algebras. A normal short complex is one

$$
\cdots \rightarrow A_{2} \rightarrow A_{1} \stackrel{\partial}{\rightarrow} A_{0}
$$

for which $A_{n}=0, n \geqslant 2$, and $\partial$ is a normal monomorphism (see [4, p. 225]). Then we have an exact sequence

$$
0 \rightarrow A_{1} \stackrel{\partial}{\rightarrow} A_{0} \stackrel{e}{\rightarrow} H_{0}(\mathrm{~A}) \rightarrow 0 .
$$

Received by the editors March 22, 1978 and, in revised form, April 20, 1979 and October 23, 1979. AMS (MOS) subject classifications (1970). Primary 55A05, 55A25, 55H05.

Key words and phrases. Group presentation, Baer invariants, spectral sequence, links.

${ }^{1}$ Died September 22, 1978. Her doctoral thesis was written at Illinois under the supervision of $K$. T. Chen. The revision presented here was made by M. A. Gutiérrez. Aside from minor changes the only significant difference with the original version lies in the treatment of the proof of Lemma 2.2 which led to a sharpening of the main theorem. Requests for reprints and comments should be directed to $\mathbf{M}$. Gutiérrez, Department of Mathematics, Tufts University, Medford, Massachusetts 02155. 
If $A_{0}$ is a projective (resp. free) algebra, then $\mathbf{A}$ is called a projective (resp. free) presentation of $H_{0}(\mathrm{~A})$. We apply this to the case where our algebra is an integral group ring.

(1.1) Let $G$ be a group; then $G_{n}$ stands for the $n$th member of its lower central series [6, Chapter $\mathrm{V}, \S 9$ ]. In particular $G_{2}$ is the commutator subgroup and $\bar{G}=G / G_{2}$ is the abelianization of $G$.

The ring $Z G$ is the integral group ring of $G$ with augmentation $\varepsilon: Z G \rightarrow Z$. Let $I G=\operatorname{ker} \varepsilon$; then $I^{n} G$ stands for the $n$th power of $I G$.

(1.2) Let now

$$
\left\langle x_{i}: r_{j}\right\rangle
$$

be the presentation [6, p. 205] for $G$. This means that we have a free group $F$ in the $x_{i}$ and that $G \cong F / R$, where $R$ is the smallest normal subgroup of $F$ generated by $\left\{r_{j}\right\} \subseteq F$. We write $R=\left\langle r_{j}\right\rangle$.

Consider the 2-sided ideal $N=\left(r_{j}-1\right)$ of $Z F$ generated by the $r_{j}-1$. Then we have a free presentation

$$
0 \rightarrow N \stackrel{\partial_{1}}{\rightarrow} Z F \rightarrow Z G \rightarrow 0
$$

of $Z G$. Since $N \subseteq I F$ we may take the short complex $[4, \S 2]$

$$
0 \rightarrow N \stackrel{\partial_{1}}{\rightarrow} I F
$$

(here $J_{q}=0, q \geqslant 2, J_{1}=N$ and $J_{0}=I F$ ), which is a free presentation of $F G$, via the isomorphism $H_{0}(J) \cong I G$, since $I F$ is $F$-free [6, Chapter VI, Theorem 5.5]. By Lemma 5.2 of [4], $I F$ is a projective algebra.

$\mathbf{J}$ can be considered to be the augmentation kernel of the complex

$$
0 \rightarrow N \stackrel{\partial_{1}}{\rightarrow} Z F
$$

and the powers $\mathbf{J}^{p}$ of $\boldsymbol{J}$ define a filtration $F_{-p} \mathbf{C}=\boldsymbol{J}^{p}$ on $\mathbf{C}$. Notice that if wodefine

$$
\begin{gathered}
N(0)=N(1)=N \text { and } \\
N(p)=N(1) I^{p-1} F+I F N(p-1)=N(p-1) I F+I F N(p-1)
\end{gathered}
$$

then $\mathbf{J}^{p}=N(p) \oplus I^{p} F$.

(1.3) The filtration $F$ induces a spectral sequence in the usual manner [6, Chapter VIII, §2]. Since our filtration degree is negative, our sequence lies in the 2nd quadrant and since $\mathbf{C}_{q}=0, q \geqslant 2$, then $E_{-s, s+k}^{r}=0$ for $k \geqslant 2$, whereas

$$
E_{-s, s}^{r}=I^{s} F /\left(I^{s+1} F+N(s-r+1) \cap I^{s} F\right), \quad s>0,
$$

and

$$
E_{-s, s+1}^{r}=\left(N(s) \cap I^{s+r} F\right) /\left(N(s+1) \cap I^{s+r} F\right), \quad s \geqslant 1 .
$$

Definition (1.4) The spectral sequence $E$ is called the spectral sequence of $G$ (associated to the presentation (P)).

2. The main theorem. Our main goal is to show that $E$ depends only on $G$.

(2.1) Let then (P) be the presentation in (1.2) and let

$$
\left\langle x_{k}^{\prime}: r_{l}^{\prime}\right\rangle
$$


be another presentation. Put $F^{\prime}=\left\langle x_{k}^{\prime}:\right\rangle$ and $R^{\prime}=\left\langle r_{l}^{\prime}\right\rangle^{\prime}$; let $E^{\prime}$ be the spectral sequence associated to $(\mathrm{Q})$.

LemMa (2.2) If there exists an epimorphism $\phi: F \rightarrow F^{\prime}$ with $\phi(R)=R^{\prime}$ then $\phi$ induces an isomorphism $\Phi: E \rightarrow E^{\prime}$ of spectral sequences.

MAIN TheORem (2.3) If (P) and (Q) are any two presentations of the group $G$ then the corresponding spectral sequences are isomorphic.

This allows us to drop the parenthetical remark in Definition (1.4).

The theorem follows from (2.2) for there exists a presentation of $G,(S)$ : $\left\langle y_{\alpha}: s_{\beta}\right\rangle$ where $L=\left\langle y_{\alpha}:\right\rangle$ and $S=\left\langle s_{\beta}\right\rangle^{L}$ and epimorphisms $\psi: L \rightarrow F$ and $\psi^{\prime}:$ $L \rightarrow F^{\prime}$ with $\psi(S)=R$ and $\psi\left(S^{\prime}\right)=R^{\prime}$.

(2.4) Now we proceed to prove (2.2). Let $(I G)^{(s)}$ be the $s$-fold tensor product of $I G$ over $G$. By [4, Lemma 5.2], $(I G)^{(s)}$ has a structure of $G$-module. We contend that

$$
E_{-s, s}^{1} \cong H_{0}\left(G,(I G)^{(s)}\right)
$$

and

$$
E_{-s, s+1}^{1} \cong H_{1}\left(G,(I G)^{(s)}\right)
$$

To show this we employ [4, Theorem 7.1]: $\mathbf{J}$ is a normal short complex (cf. [4, §2]) and $H_{0}(\mathrm{~J}) \cong I G$ is a projective presentation of $I G$. Notice that $\mathrm{J}_{0}=I F$ and by formula (6.3) of [4], $V_{1}^{s}(\mathrm{~J})$ is defined by (1) and so $V_{1}^{s}(J)=N(s)$. Then by formulae (6.6) (loc. cit.),

$$
\operatorname{Tor}_{0}^{G}\left((I G)^{(s)}, Z\right)=I^{s} F /\left(I^{s+1} F+N(s)\right)
$$

and

$$
\operatorname{Tor}_{1}^{G}\left((I G)^{(s)}, Z\right)=\left(I^{s+1} F \cap N(s)\right) / N(s+1)
$$

which in view of (2) and (3) prove our claim. Now, if (P) and (Q) are presentations and $\phi: F \rightarrow F^{\prime}$ the epimorphism of the hypothesis, it induces an automorphism $\phi^{\prime}$ of $G$ and by [4, Lemma 5.2] an automorphism $\phi^{(s)}$ of $(I G)^{(s)}$. Then $\Phi_{-s, t}^{\prime}: E_{-s, t}^{1} \rightarrow$ $E_{-s, t}^{\prime \prime}$ is an isomorphism for all $t$ : for $t=s$ and $s+1$ by (4) and (5) and for $t \geqslant s+2$ because both sides are trivial. The induced map is natural by definition and it commutes with the differentials. By construction $E^{2}=H\left(E^{1}, d^{\prime}\right)$ so that $\Phi: E^{2} \rightarrow E^{\prime 2}$ is an isomorphism as well. By induction $E^{r}=E^{\prime r}$ for all $r$. Q.E.D.

(2.5) We proceed to describe the terms $E_{-s, s}^{1}$ and $E_{-1,2}^{1}$ : for $E_{-s, s}^{1}$ we employ (4)

$$
\begin{aligned}
H_{0}\left(G,(I G)^{(s)}\right) & =\left[I G \otimes_{G} \cdots \otimes_{G} I G\right] \otimes_{G} Z \\
& =\left[I G \otimes_{G} \cdots \otimes_{G} I G\right] \otimes_{G}\left(I G \otimes_{G} Z\right)
\end{aligned}
$$

where the first brackets enclose an $s$-fold product and the second enclose an $(s-1)$-fold product. By [6, Chapter VI, Lemma 4.1] IG $\otimes_{G} Z=\bar{G}$ which is a 
trivial $G$-module. Thus

$$
\begin{aligned}
{\left[I G \otimes_{G} \cdots \otimes_{G} I G\right] \otimes_{G} \bar{G} } & =\left[I G \otimes_{G} \cdots \otimes_{G} I G\right] \otimes_{G}\left(I G \otimes_{G} \bar{G}\right) \\
& =\left[I G \otimes_{G} \cdots \otimes_{G} I G\right] \otimes_{G}\left(I G \otimes_{G}\left(Z \otimes_{Z} \bar{G}\right)\right) \\
& =\left[I G \otimes_{G} \cdots\right] \otimes_{G}\left(\left(I G \otimes_{G} Z\right) \otimes_{Z} \bar{G}\right) \\
& =\left[I G \otimes_{G} \cdots\right] \otimes_{G}\left(\bar{G} \otimes_{Z} \bar{G}\right) .
\end{aligned}
$$

By succesive applications of this we get

LEMMA (2.6) $E_{-s, s}^{1}$ is the s-fold tensor product of $\bar{G}$ over $Z$.

REMARK. In the notation of $[4, \S 5], E_{-s, s}^{1}=\bar{G}^{(s)}$.

LeMMA (2.7) $E_{-1,2}^{1}=H_{2}(G ; Z)$.

Proof. $E_{-1,2}^{1}=H_{1}(G, I G)=H_{2}(G ; Z)$ by [6, Chapter VI, Theorem 12.1].

(2.8) In our thesis we worked out an explicit isomorphism $E_{-s, s}^{1} \rightarrow \bar{G}^{(s)}$ as follows: $\bar{G}$ is naturally isomorphic to $I F /\left(N+I^{2} F\right)$. Consider

$$
\gamma:\left(I F /\left(N+I^{2} F\right)\right)^{(s)} \rightarrow I^{s} F /\left(N(s)+I^{s+1} F\right)
$$

defined by

$$
\gamma\left(\overline{\left(x_{i_{1}}-1\right)} \otimes \cdots \otimes \overline{\left(x_{i_{s}}-1\right)}\right)=\prod\left(x_{i_{j}}-1\right)+\left(N(s)+I^{s+1} F\right) .
$$

If $\Phi_{-1,1}^{\prime}:\left(I F / N+I^{2} F\right) \rightarrow\left(I F^{\prime} / N^{\prime}+I^{2} F^{\prime}\right)$ is the isomorphism defined by $\phi$ (and $\left.N^{\prime}=\left(r_{l}^{\prime}-1\right) \subseteq Z F^{\prime}\right)$ then

$$
\Phi_{-s, s}^{\prime} \gamma=\left(\Phi_{-1,1}^{\prime}\right)^{(s)} \gamma^{\prime}
$$

Similarly, if $h: F \rightarrow Z F$ is a map $x \mapsto x-1$ then $h$ induces an isomorphism $\left(R \cap F_{2}\right) /[F, R] \rightarrow\left(N \cap I^{2} F\right) / N(2)$ and the former quotient is the well-known Hopf formula for $H_{2}(G ; Z)[6$, p. 204]. We omit the proofs.

Proposition (2.9) $E_{-s, s}^{s}=E_{-s, s}^{\infty}=I^{s} G / I^{s+1} G$.

Proof. Since $Z G \cong Z F / N, I G=I F / N$. Consider

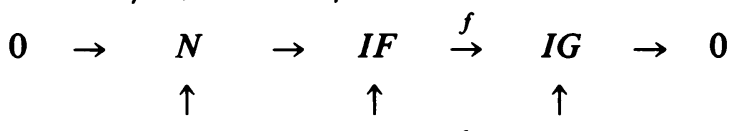

$$
\begin{aligned}
& 0 \rightarrow \operatorname{ker} h \quad \rightarrow \quad I^{s} F \stackrel{h}{\rightarrow} \quad I^{s} G \quad \rightarrow \quad 0
\end{aligned}
$$

where $h=f \mid I^{s} F$ then $\operatorname{ker} h=\operatorname{ker} f \cap I^{s} F=N \cap I^{s} F$. Hence

$$
I^{s}(G) \simeq I^{s} F /\left(N \cap I^{s}\right) \simeq\left(N+I^{s} F\right) / N .
$$

By the Noetherian isomorphism theorem,

$$
\begin{aligned}
I^{s+1} F & \subset I^{s} F, E_{-s, s}^{s} \cong I^{s} F /\left(I^{s} F \cap N+I^{s+1} F\right) \\
& =\frac{I^{s} F /\left(I^{s} F \cap N\right)}{\left(I^{s} F \cap N+I^{s+1} F\right) /\left(I^{s} F \cap N\right)} \cong \frac{I^{s} F /\left(I^{s} F \cap N\right)}{I^{s+1} F /\left(I^{s} F \cap N \cap I^{s+1} F\right)} \\
& =\frac{I^{s} F /\left(I^{s} F \cap N\right)}{I^{s+1} F /\left(I^{s+1} F \cap N\right)}=I^{s} G / I^{s+1} G .
\end{aligned}
$$


LEMMA (2.10) If $g$ is an element of $G_{n}$ then $g-1$ is an element in $I^{n} G$ for all $n \geqslant 1$.

TheOREM (2.11) Let $\bar{E}$ be the spectral sequence of the group $\Gamma=G / G_{q+1} ; q$ is any integer $\geqslant 1$. Let $E$ be the spectral sequence of $G$. Then

$$
\begin{gathered}
E_{-r, r}^{r} \simeq \bar{E}_{-r, r}^{r} \quad \text { for } 1 \leqslant r<q, \\
E_{-s, s}^{r} \simeq \bar{E}_{-s, s}^{r} \text { for } 1<s<r \leqslant q .
\end{gathered}
$$

Proof. Statement (7) follows from (6) because

$$
E_{-s, s}^{r} \simeq \cdots \simeq E_{-s, s}^{s+1} \simeq E_{-s, s}^{s} \simeq \bar{E}_{-s, s}^{s} \simeq \bar{E}_{-s, s}^{s+1} \simeq \cdots \simeq \bar{E}_{-s, s}^{r} .
$$

To prove (6) it is enough to show that

$$
I G / I^{r+1} G \simeq I \Gamma / I^{r+1} \Gamma \text { for } r<q .
$$

The canonical epimorphism $G \rightarrow G / G_{q+1}$ induces the ring epimorphism $Z G \rightarrow$ $Z \Gamma$. Define

$$
\phi: I G \rightarrow I \Gamma / I^{r+1} \Gamma \quad \text { by } g-1 \rightarrow g^{\prime}-1+I^{r+1} \Gamma,
$$

where $g^{\prime}=g G_{q+1}$. Since $\phi\left(I^{r+1} G\right)=I^{r+1} \Gamma, \phi$ induces the epimorphism

$$
\Phi: I G / I^{r+1} G \rightarrow I \Gamma / I^{r+1} \Gamma
$$

given by

$$
g-1+I^{r+1}(G) \rightarrow g^{\prime}-1+I^{r+1} \Gamma .
$$

But $g-1+I^{r+1} G$ generates $I G / I^{r+1} G$. Finally, we define an inverse to $\Phi$. Define

$$
\psi: I \Gamma \rightarrow I G / I^{r+1} G, \quad g^{\prime}-1 \rightarrow g-1+I^{r+1} G,
$$

where $g^{\prime}=g G_{q+1}$. The map $\psi$ is well defined, for if $g^{\prime}=h^{\prime}$, then $h=g w$, where $w \in G_{q+1}$, but $g w-1=(g-1)(w-1)+(g-1)+(w-1)$ and by Lemma (2.10), w- $w \in I^{q+1} G \subset I^{r+1} G$, since $r<q$ and $(g-1)(w-1) \in I^{q+2} G \subset$ $I^{r+1} G$. Therefore, $\psi\left(h^{\prime}-1\right)=(g w-1)+I^{r+1} G=(g-1)+I^{r+1} G$. Consider the composite map, $I G \rightarrow I \Gamma \rightarrow I G / I^{r+1} G$, this is a ring homomorphism, and it carries $I^{r+1} G \rightarrow I^{r+1} \Gamma \rightarrow 0$. Therefore $\psi$ induces

$$
\psi: I \Gamma / I^{r+1} \Gamma \rightarrow I G / I^{r+1} G .
$$

But $\psi \circ \Phi=1$ and $\Phi \circ \psi=1$; hence the result.

REMARKS. (1) In the course of the proof of Theorem (2.11) we have shown that

$$
\Phi: I G / I^{n} G \stackrel{\sim}{\rightarrow} I \Gamma / I^{n} \Gamma
$$

where $\Gamma=G / G_{n}($ see (8)).

(2) Let $E$ be the sequence of $G$ associated to the presentation (P) as defined in (1.4), and let $K$ be an Eilenberg-Mac Lane space of type $(G, 1)$. If $\Lambda^{p} K$ denotes the $p$-fold smash product [9] of $K$ with itself, then the formula $\bar{E}_{-p, q}^{1}=H_{q}\left(\Lambda^{p} K\right)$ describes a spectral sequence $\bar{E}$ whose 1 -skeleton is described in $[5, \S 1]$ and $[9, \S 3]$. Since $\bar{E}_{-p, p}^{1}$ is isomorphic to $E_{-p, p}^{1}$ and since $\bar{E}^{\infty}$ is isomorphic to $E^{\infty}$, there is a natural map $\bar{E}^{1} \rightarrow E^{1}$. This map, however, is not monic because the terms $\bar{E}_{-p, p+k}^{1}$ $(k \geqslant 2)$ are not zero while the corresponding terms in $E$ are. The map, on the other hand, is onto. 
3. Applications to links. Let $S^{(n)}$ be the space consisting of $n$-disjoint circles $S_{1}, \cdots, S_{n}$. Assume that fixed orientations have been chosen for $S^{(n)}$ and $R^{3}$. By an oriented $n$-link $l$ in $R^{3}$ is meant a homeomorphic image of $S^{(n)}$ in $R^{3}$. Thus $l$ can be thought of as an ordered collection $\left(l_{1}, l_{2}, \ldots, l_{n}\right)$ of homeomorphisms $l_{i}$ : $S_{i} \rightarrow R^{3}$; where the images $L_{1}, L_{2}, \ldots, L_{n}$ of the $l_{i}$ 's are to be disjoint. Denote $L_{1} \cup L_{2} \cup \cdots \cup L_{n}$ by $L$, and the fundamental group of the complement of $L$ in $R^{3}, \pi\left(R^{3}-L, x_{0}\right)$ by $G(L)$, where $x_{0} \in R^{3}-L$ is a fixed chosen base point. Let $N_{1}, N_{2}, \ldots, N_{n}$ be solid tori chosen to be regular, disjoint neighborhoods of $L_{1}, L_{2}, \ldots, L_{n}$ respectively. Let $p_{i}(t)(0 \leqslant t \leqslant 1)$ be a path from the point $x_{0}$ to a point on the boundary of $N_{i}$. A meridian-longitude pair $\left(\alpha_{i}, \beta_{i}\right)$ for $L$ is a pair of elements of $G(L)$ where:

(i) $\alpha_{i}$ is represented by a closed loop in $R^{3}-L$ described as follows: traverse $p_{i}$, then traverse a closed loop on the boundary of $N_{i}-L_{i}$ which has linking number +1 with $l_{i}$ and finally return to $x_{0}$ along $p_{i}$;

(ii) $\beta_{\mathrm{i}}$ is represented by a closed loop in $R^{3}-L$ described as follows: traverse $p_{i}$, then traverse a simple closed curve on the boundary of $N_{i}$ which has linking number 0 with $l_{i}$ and which is nullhomologous in $R^{3}-L_{i}$, and finally return to $x_{0}$ along $p_{i}$.

The elements $\alpha_{i}, \beta_{i}$ of $G(L)$ are well defined in $G(L)$ up to the choice of $p_{i}$ and the orientations chosen for $S^{(n)}$ and $R^{3}$. Any other $i$ th meridian-longitude pair $\left(\alpha_{i}^{\prime}, \beta_{i}^{\prime}\right)$ for $L$ is obtained from $\left(\alpha_{i}, \beta_{i}\right)$ by simultaneous conjugation, that is, $\alpha_{i}^{\prime}=g \alpha_{i} g^{-1}$ and $\beta_{i}^{\prime}=g \beta_{i} g^{-1}$ for some $g \in G(L)$.

Two links $l$ and $l^{\prime}$ are said to be isotopic if there exists a continuous family $h_{t}$ : $S^{(n)} \rightarrow R^{3}$ of homeomorphisms, for $0 \leqslant t \leqslant 1$, with $h_{0}=l$ and $h_{1}=l^{\prime}$. The fundamental group $G(L)$ of the complement of $L$ in $R^{3}$ is not invariant under isotopy of the link. In 1952, K. T. Chen proved [2] that $G(L) / G_{q}(L)$, where $G_{q}(L)$ is the qth lower central subgroup of $G(L)$, is invariant under isotopy of the link for any arbitrary posititive integer $q$. In 1957, Milnor gave [7] a presentation describing the group $G(L) / G_{q}(L)$ and defined the so-called Milnor invariants for a link.

It is known that: if $G$ is the fundamental group of the complement of an $n$-link $l$ in $R^{3}$ then $G / G_{2}$ is free abelian of rank $n$.

In Theorem (2.11) we found that if $\bar{E}$ is the spectral sequence of $G / G_{s+1}, s>1$, and $E$ is the spectral sequence of $G$ that then $E_{-s, s}^{s} \simeq \bar{E}_{-s, s}^{s}$. In the light of the above stated result of Chen we can conclude:

THEOREM (3.1) Let $G$ be the group of a certain $n$-link $l$. Let $E$ be the spectral sequence of $G / G_{s+1}$. Then $E_{-s, s}^{s}$ is an isotopy invariant of the link $l$.

Let $\left\langle a_{i j}: r_{i j}\right\rangle\left(i=1,2, \ldots, n ; j=1, \ldots, k_{i}\right)$ be a Wirtinger presentation for $G(L)$ (henceforth we shall write $G$ for $G(L)$ ) where to each crossing point $Q_{i j}$ of the projection corresponds a relation $r_{i j}=1, r_{i j}=\left[b_{i j}, a_{i j}\right] a_{i j} a_{i j+1}^{-1}$ with $b_{i j}=a_{\lambda}^{\ell_{i j}(i) \mu(i j)}$, $(\lambda(i j), \mu(i j))$ are given by the segment of $L$ which crosses over at $Q_{i j}$, and $\varepsilon_{i j}= \pm 1$ is the signature of the crossing. Let $v_{i j}=\left[b_{i j}, a_{i j}\right]$ and $a_{i 1}=a_{i}$. Define

$$
u_{i 1}=1 \text { and } u_{i j}=v_{i j-1} v_{i j-2} \cdots v_{i 1} \quad\left(j=2,3, \ldots, k_{i}\right)
$$


and

$$
w_{i k_{i}}=b_{i 1}^{-1} b_{i 2}^{-1} \cdots b_{i k_{i}}^{-1}
$$

Then $G$ may be presented by

$$
\begin{gathered}
\left\langle a_{i j}: h_{i j}, s_{i}\right\rangle \quad\left(i=1, \ldots, n ; j=1,2, \ldots, k_{i}\right), \\
h_{i 1}=1, \quad h_{i j}=u_{i j} a_{i} a_{i j}^{-1} \quad\left(j=2, \ldots, k_{i}\right), \\
s_{i}=\left[a_{i}, w_{i k_{i}}\right] .
\end{gathered}
$$

Note, $w_{i k_{i}}$ is an $i$ th longitude of $L$ in $G$. Thus $Z G \simeq Z F / N$ where $F$ is the free group on the $a_{i j}$ 's and $N$ is the ideal of $Z F$ generated by $h_{i j}-1, s_{i}-1$ ( $i=$ $\left.1, \ldots, n ; j=2, \ldots, k_{i}\right)$. Since $h_{i j}-1=\left(u_{i j}-a_{i j} a_{i}^{-1}\right) a_{i} a_{i j}^{-1}$ and $a_{i} a_{i j}^{-1}$ is a unit of $Z F, N$ is generated as an ideal of $Z F$ by

$$
\left\{u_{i j}-a_{i j} a_{i}^{-1}, s_{i}-1\right\} \quad\left(i=1, \ldots, n ; j=2, \ldots, k_{i}\right) .
$$

LEMMA (3.2) Let $N_{1}$ be the ideal of $Z F$ generated by $\left\{u_{i j}-a_{i j} a_{i}^{-1}\right\}(i=1, \ldots, n$; $\left.j=2, \ldots, k_{i}\right)$. Then

$$
N_{1} \cap I^{s} F=N_{1}(s),
$$

where $I F=\operatorname{ker}(Z F \rightarrow Z), N_{1}(1)=N_{1}$, and $N_{1}(s)=I F N_{1}(s-1)+N_{1}(s-1) I F$ $(s>1)$.

Proof. The elements $\left\{u_{i j}-a_{i j} a_{i}^{-1}+N_{1}(2)\right\}$ generate the $Z$-module $N_{1} / N_{1}(2)$. Moreover we shall show that $\left\{u_{i j}-a_{i j} a_{i}^{-1}+N_{1}(2)\right\}$ forms a basis for $N_{1} / N_{1}(2)$. Indeed, if for some integers $n_{i j}, \sum n_{i j}\left(u_{i j}-a_{i j} a_{i}^{-1}\right)=0+N_{1}(2)$, where the summation is over $i=1, \ldots, n$ and $j=2, \ldots, k_{i}$. Then $\sum n_{i j}\left(u_{i j}-a_{i j} a_{i}^{-1}\right) \in N_{1}(2)$, hence $\sum n_{i j}\left(u_{i j}-a_{i j} a_{i}^{-1}\right) \in I^{2} F$. Thus

$$
\left(\partial / \partial a_{s t}\right) \sum n_{i j}\left(u_{i j}-a_{i j} a_{i}^{-1}\right)(1)=0 \quad \text { (cf. [3]). }
$$

But $u_{i j} \in F_{2}$, (9), hence $u_{i j}-1 \in I^{2}$, so, $\left(\partial / \partial a_{s t}\right)\left(u_{i j}-1\right)(1)=0$ and $\partial a_{i j} / \partial a_{s t}=0$ if $(i, j) \neq(s, t)$ and $\partial a_{s t} / \partial a_{s t}=1$. Therefore,

$$
\begin{aligned}
\left(\partial / \partial a_{s t}\right) \sum n_{i j}\left(u_{i j}-a_{i j} a_{i}^{-1}\right)(1) & =\left(\partial / \partial a_{s t}\right) \sum n_{i j}\left(u_{i j}-1-a_{i j} a_{i}^{-1}+1\right)(1) \\
& =\sum-n_{i j}\left(\partial / \partial a_{s t}\right)\left(a_{i j} a_{i}^{-1}\right)(1) \\
& =\sum-n_{i j}\left(\left(\partial / \partial a_{s t}\right) a_{i j}(1)+a_{i j}(1)+a_{i j}\left(\partial / \partial a_{s t}\right) a_{i}^{-1}(1)\right) \\
& =-n_{s t} .
\end{aligned}
$$

Hence $n_{s t}=0$ (see (14)).

Thus the sequence of $Z$-modules

$$
0 \rightarrow N_{1}(2) \rightarrow N_{1} \rightarrow N_{1} / N_{1}(2) \rightarrow 0,
$$

is split exact. Let $M$ be the $Z$-submodule of $N_{1}$ generated by $\left\{u_{i j}-a_{i j} a_{i}^{-1}\right\}$ $\left(i=1, \ldots, n ; j=2, \ldots, k_{i}\right)$. Then $N_{1}=M+N_{1}(2)$. Since

$$
\left(\partial / \partial a_{i j}\right)\left(u_{i j}-a_{i j} a_{i}^{-1}\right)(1)=-1, \quad u_{i j}-a_{i j} a_{i}^{-1} \in I F,
$$

but not in $I^{2} F$. So $M \cap I^{2} F=\{0\}$, and

$$
N_{1} \cap I^{2} F=N_{1}(2) \text {. }
$$


But

$$
N_{1}(s+1)=\sum_{i=1}^{s-1} I^{i} F N_{1}(2) I^{s-i-1} F
$$

and

$$
N_{1}(s) \cap I^{s+1} F=\sum_{i=0}^{s-1} I^{i} F\left(N_{1} \cap I F\right) I^{s-i-1} F
$$

Therefore

$$
N_{1}(s+1)=\sum_{i=0}^{s-1} I^{i} F\left(N_{1} \cap I^{2} F\right) I^{s-i-1} F=N_{1}(s) \cap I^{s+1} F
$$

The proof of (13) follows from (15) by induction on $s$.

Let $N_{2}$ be the ideal of $Z F$ generated by $\left\{s_{i}-1\right\}(i=1, \ldots, n)$, then one can write $N=N_{1}+N_{2}, N_{1}$ is the $Z F$-ideal generated by $\left\{u_{i j}-a_{i j} a_{i}^{-1}\right\}(i=1, \ldots, n$; $j=2, \ldots, k_{i}$ ) (see Lemma (3.2)).

LEMMA (3.3) If $s_{i}-1$ is in $I^{s} F$ for $i=1, \ldots, n$, then

$$
E_{-s, s}^{s-1} \simeq E_{-s, s}^{s-2} \simeq \cdots \simeq E_{-s, s}^{1} \simeq \otimes^{s} I F /\left(N+I^{2} F\right) .
$$

Proof. By (2) and (3),

$$
E_{-s, s}^{r}=I^{s} F /\left(N(s-r+1) \cap I^{s} F+I^{s+1} F\right) .
$$

Let $t=s-r+1$, then $2 \leqslant t \leqslant s$. Now $N(t)=N_{1}(t)+N_{2}(t)$, where $N_{2}$ is the ideal of $Z F$ generated by $s_{i}-1$; hence $N_{2} \subset I^{s} F$. So, $N(t) \cap I^{s} F=N_{2}(t)+N_{1}(t)$ $\cap I^{s} F$. But $N_{1}(t)=N_{1} \cap I^{t} F$ (see (13)). Therefore $N_{1}(t) \cap I^{s} F=N_{1} \cap I^{s} F$. Since $N_{2} \subset I^{s} F, N_{2}(t) \subset I^{s+1} F$. Hence for $1<r<s-1, N(s-r+1) \cap I^{s} F+I^{s+1} F$ $=N_{1} \cap I^{s} F+I^{s+1} F=N_{1}(s)+I^{s+1} F$; the last equality follows from (13). Therefore

$$
I^{s} F /\left(N_{1}(s)+I^{s+1} F\right) \simeq E_{-s, s}^{s-1} \simeq E_{-s, s}^{s-2} \simeq \cdots \simeq E_{-s, s}^{1} \simeq \bigotimes^{s} I F /\left(N+1^{2} F\right) .
$$

Corollary (3.4) If $s_{i}-1$ is in $I^{s} F$ for $(i=1, \ldots, n)$ then $E_{-s, s}^{r}(1<r<s-1)$ is free abelian of rank $n^{s}$.

Proof. This follows from the fact that $G / G_{2}$ is free abelian of rank $n$, Lemma (3.3) and the isomorphism $I / N+I^{2} \simeq G / G_{2}$.

Next we shall describe a basis for $E_{-s, s}^{r}=I^{s} F /\left(N_{1}(s)+I^{s+1} F\right)(1<r<s-1)$. Here again we assume that $s_{i}-1 \in I^{s} F, i=1, \ldots, n$.

Recall that $N_{1}$ is the ideal of $Z F$ generated by $\left\{u_{i j}-a_{i j} a_{i}^{-1}\right\}(i=1,2, \ldots, n$; $\left.j=2,3, \ldots, k_{i}\right)$. Let $\eta_{i j}-a_{i j} a_{i}^{-1}$ and $x_{i}=a_{i}-1$. Then

$$
\begin{aligned}
\eta_{i j} & =u_{i j}-1-a_{i j} a_{i}^{-1}+1 \\
& =\left(a_{i}-1\right)-\left(a_{i j}-1\right)+\left(u_{i j}-1\right)-\left(a_{i j}-1\right)\left(a_{i}^{-1}-1\right)+\left(a_{i}^{-1}-1\right) .
\end{aligned}
$$

Let $W_{i j}=\left(u_{i j}-1\right)-\left(a_{i j}-1\right)\left(a_{i}^{-1}-1\right)+\left(a_{i}-1\right)\left(a_{i}^{-1}-1\right)$. Then $W_{i j} \in I^{2} F$. Hence

$$
\left\{\begin{array}{l}
a_{i j}=1+\chi_{i}+W_{i j}+\eta_{i j} \\
a_{i j}^{-1}=1-\chi_{i}-W_{i j}^{\prime}-\eta_{i j}
\end{array}\right.
$$


where $W_{i j}^{\prime}=W_{i j}+\left(a_{i j}-1\right)\left(a_{i j}^{-1}-1\right) \in I^{2} F$ and $\eta_{i j} \in N_{1}$.

Since $G \simeq F / R$, where $F$ is the free group on $\left\{a_{i j}: i=1, \ldots, n ; j=1, \ldots, k_{i}\right\}$, the set $\left\{\left(a_{i_{1} j_{1}}-1\right)\left(a_{i_{2} j_{2}}-1\right) \cdots\left(a_{i_{s} j_{s}}-1\right)+N_{1}(s)+I^{s+1} F\right\} \quad\left(i_{1}, i_{2}, \ldots, i_{s}=\right.$ $1, \ldots, n$ and $\left.j_{1}, j_{2}, \ldots, j_{s}=1,2, \ldots, k_{i}\right)$ generates $I^{s} F /\left(N_{1}(s)+I^{s+1} F\right)$. Using the equalities (17) one can write

$$
\prod_{t=1}^{s}\left(a_{i, j_{t}}-1\right)+N_{1}(s)+I^{s+1} F=\prod_{t=1}^{s} \chi_{i_{i}}+N_{1}(s)+I^{s+1} F
$$

Thus,

$$
\begin{array}{r}
\left\{\left(a_{i_{1}}-1\right)\left(a_{i_{2}}-1\right), \ldots,\left(a_{i_{s}}-1\right)+N_{1}(s)+I^{s+1} F\right\} \\
\left(i_{1}, \ldots, i_{s}=1, \ldots, n\right)
\end{array}
$$

forms a generating set of $I^{s} /\left(N_{1}(s)+I^{s+1}\right)$. But there are $n^{s}$ elements in the set (18); hence (18) forms a $Z$-basis for $I^{s} /\left(N_{1}(s)+I^{s+1}\right)$ (see Corollary 3.4).

Consider the $E^{s-1}$ term of the spectral sequence $E$,

$$
\rightarrow E_{s-2,4-s}^{s-1} \rightarrow E_{-1,2}^{s-1} \stackrel{d_{-1,2}^{s-1}}{\rightarrow} E_{-s, s}^{s-1} \stackrel{d_{s, s}^{s-1}}{\rightarrow} E_{-2 s+1,2 s-2} \rightarrow \cdots,
$$

where all terms of degree $\neq 0,1$ of $E^{s-1}$ are zero. Therefore we have

$$
\rightarrow 0 \rightarrow E_{-1,2}^{s-1} \stackrel{d_{-1,2}^{s-1}}{\rightarrow} E_{-s, s}^{s-1} \stackrel{d_{-s-s}^{s-1}}{\rightarrow} 0
$$

Explicitly, we have

$$
\rightarrow 0 \rightarrow\left(N \cap I^{s} F\right) /\left(N(2) \cap I^{s} F\right) \stackrel{d_{-1,2}^{s-1}}{\rightarrow} I^{s} F /\left(I^{s+1} F+N(2) \cap I^{s} F\right) \stackrel{d_{s, s}^{s-1}}{\rightarrow} 0,
$$

where $d_{-1,2}^{s-1}$ is induced from the inclusion $N \cap I^{s} F \rightarrow I^{s} F$. But

$$
\begin{aligned}
E_{-s, s}^{s} & \simeq H\left(E_{-s, s}^{s-1}\right) \simeq \operatorname{ker} d_{-s, s}^{s-1} / d_{-1,2}^{s-1}\left(E_{-1,2}^{s-1}\right) \\
& \simeq \frac{I^{s} F /\left(N(2) \cap I^{s} F+I^{s+1} F\right)}{\left(N \cap I^{s} F+N(2) \cap I^{s} F+I^{s+1} F\right) /\left(N(2) \cap I^{s} F+I^{s+1} F\right)} \\
& \simeq \frac{I^{s} F /\left(N(2) \cap I^{s} F+I^{s+1} F\right)}{\left(N \cap I^{s} F+I^{s+1} F\right) /\left(N(2) \cap I^{s} F+I^{s+1} F\right)},
\end{aligned}
$$

since $N(2) \cap I^{s} F \subset N \cap I^{s} F$.

TheOREM (3.5) If $s_{i}-1 \in I^{s} F(i=1,2, \ldots, n)$, then

$$
E_{-s, s}^{s} \simeq \frac{I^{s} F /\left(N_{1}(s)+I^{s+1} F\right)}{\left(N_{2}+N_{1}(s)+I^{s+1} F\right) /\left(N_{1}(s)+I^{s+1} F\right)},
$$

where the set $\left\{\left(a_{i_{1}}-1\right)\left(a_{i_{2}}-1\right) \cdots\left(a_{i_{s}}-1\right)+N_{1}(s)+I^{s+1} F\right\}\left(i_{1}, i_{2}, \ldots, i_{s}=\right.$ $1, \ldots, n)$, gives a basis for $I^{s} F /\left(N_{1}(s)+I^{s+1} F\right)$, and where the set $\left(s_{i}-1\right)+N_{1}(s)$ $+I^{s+1}(i=1, \ldots, n)$, gives a basis for $\left(N_{2}+N_{1}(s)+I^{s+1} F\right) /\left(N_{1}(s)+I^{s+1} F\right)$.

Proof. Since $s_{i}-1 \in I^{s} F, N_{2} \subset I^{s} F$. Hence $N \cap I^{s} F=N_{1} \cap I^{s} F+N_{2}=N_{1}(s)$ $+N_{2}$ (see (13)). Also since $N(2)=N_{1}(2)+N_{2}(2)$ and $N_{2}(2) \subset I^{s+1} F$, it follows that

$$
N(2) \cap I^{s} F=N_{1}(2) \cap I^{s} F=N_{1} \cap I^{2} F \cap I^{s} F=N_{1} \cap I^{s} F=N_{1}(s) .
$$


Substituing these equalities in (19) we get (20). The rest of Theorem (3.5) is clear.

Since

$$
\begin{aligned}
s_{i}-1 & =\left[a_{i}, w_{i k_{i}}\right]-1=\left(a_{i} w_{i k_{i}}-w_{i k_{i}} a_{i}\right) a_{i}^{-1} w_{i k_{i}}^{-1} \\
& =\left(\left(a_{i}-1\right)\left(w_{i k_{i}}-1\right)-\left(w_{i k_{i}}-1\right)\left(a_{i}-1\right)\right) a_{i}^{-1} w_{i k_{k}}^{-1} .
\end{aligned}
$$

Hence $N_{2}$ may be thought of as being generated by $\left\{\chi_{i}\left(w_{i k_{i}}-1\right)-\left(w_{i k_{i}}-1\right) \chi_{i}\right.$ : $i=1, \ldots, n\}$. Thus, as a $Z$-module, $\left(N_{2}+N_{1}(s)+I^{s+1} F\right) /\left(N_{1}(s)+I^{s+1} F\right)$ is generated by $\left\{\chi_{i}\left(w_{i k_{i}}-1\right)-\left(w_{i k_{i}}-1\right) \chi_{i}+N_{1}(s)+I^{s+1} F\right\}$.

A simple computation shows that for any $n$-link,

$$
s_{i}-1=\sum_{j=1}^{n} \mu(i, j)\left(\chi_{i} \chi_{j}-\chi_{j} \chi_{i}\right)+N_{1}(2)+I^{3} F
$$

where $\mu(i, j)$ is the linking number of the $i$ th and $j$ th components of $L$. Hence $E_{-2,2}^{2}$ gives very little information about $L$.

Next, we give an example where we compute $E_{-3,3}^{3}$ for a link whose $s_{i}$ 's belong to $I^{3} F$. The link is shown in the figure and one has

$$
\begin{array}{ll}
b_{12 j-1}=a_{34 j-3}, & b_{12 j}=a_{34 j}^{-1}, \\
b_{22 j-1}=a_{34 j-4}^{-1}, & b_{22 j}=a_{34 j-1}, \\
b_{34 j-3}=a_{12 j}, & b_{34 j-2}=a_{22 j}, \\
b_{34 j-1}=a_{12 j}^{-1}, & b_{34 j}=a_{22 j+2}^{-1}
\end{array} .
$$

Computing $w_{12 m}, w_{22 m}$ and $w_{34 m}$ we get

$$
\begin{aligned}
& w_{12 m}=a_{31}^{-1}\left(\left[a_{34}, a_{24}^{-1}\right]\left[a_{38}, a_{26}^{-1}\right] \cdots\left[a_{32 m}, a_{22}^{-1}\right]\right) a_{31}, \\
& w_{22 m}=a_{32 m}\left(\left[a_{33}^{-1}, a_{12}^{-1}\right]\left[a_{37}^{-1}, a_{14}^{-1}\right] \cdots\left[a_{34 m}^{-1}, a_{12 m}^{-1}\right]\right) a_{32 m}^{-1},
\end{aligned}
$$

and

$$
w_{34 m}=a_{22}^{-1}\left(\left[a_{22}, a_{12}^{-1}\right] \cdots\left[a_{22 j}, a_{12 j}^{-1}\right] \cdots\left[a_{22 m}, a_{12 m}^{-1}\right]\right) a_{32} .
$$

Hence,

(i) $s_{1}=\left[a_{1}, a_{3}^{-1}\left(\Pi_{j=1}^{m}\left[a_{34 j}, a_{22 j+2}^{-1}\right]\right) a_{3}\right]$,

(ii) $s_{2}=\left[a_{2}, a_{32 m}\left(\Pi_{j=1}^{m}\left[a_{34-1}^{-1}, a_{12 j}^{-1}\right]\right) a_{32 m}^{-1}\right]$,

(iii) $s_{3}=\left[a_{3}, a_{22}^{-1}\left(\Pi_{j=1}^{m}\left[a_{22 j}, a_{12 j}^{-1}\right]\right) a_{22}\right]$.

Upon making use of the substitutions (17) for the different $a_{i j}$ and $a_{i j}^{-1}$ we obtain

$$
\begin{aligned}
& s_{1}-1=m\left[X_{1},\left[X_{2}, X_{3}\right]\right]+N_{1}(3)+I^{4} F, \\
& s_{2}-1=m\left[X_{2},\left[X_{3}, X_{1}\right]\right]+N_{1}(3)+I^{4} F, \\
& s_{3}-1=m\left[X_{3},\left[X_{1}, X_{2}\right]\right]+N_{1}(3)+I^{4} F,
\end{aligned}
$$

where by $[X, Z]$ we mean the usual Lie bracket, $[X, Z]=X Z-Z X$. Thus $\left(N_{2}+N_{1}(3)+I^{4} F\right) /\left(N_{1}(3)+I^{4} F\right)$ is generated by $s_{1}-1, s_{2}-1$ and $s_{3}-1$ as in Theorem (3.5). But $\left[X_{1},\left[X_{2}, X_{3}\right]\right]+\left[X_{2},\left[X_{3}, X_{1}\right]\right]+\left[X_{3},\left[X_{1}, X_{2}\right]\right]=0$ so that $s_{1}-$ 1 and $s_{2}-1$ form a basis for $\left(N_{2}+N_{1}(3)+I^{4} F\right) /\left(N_{1}(3)+I^{4} F\right)$. Therefore

$$
E_{-3,3}^{3} \simeq Z \oplus \cdots \oplus Z \oplus Z_{m} \oplus Z_{m},
$$


where there are twenty-five copies of $Z$ in the above sum; since

$$
\left(I^{s} F\right) /\left(N_{1}(s)+I^{s+1}\right) \simeq Z \oplus \cdots \oplus Z,
$$

there are twenty-seven copies of $Z$. Thus 3-links of the type shown in the figure whose $m$ 's differ are distinguishable links.

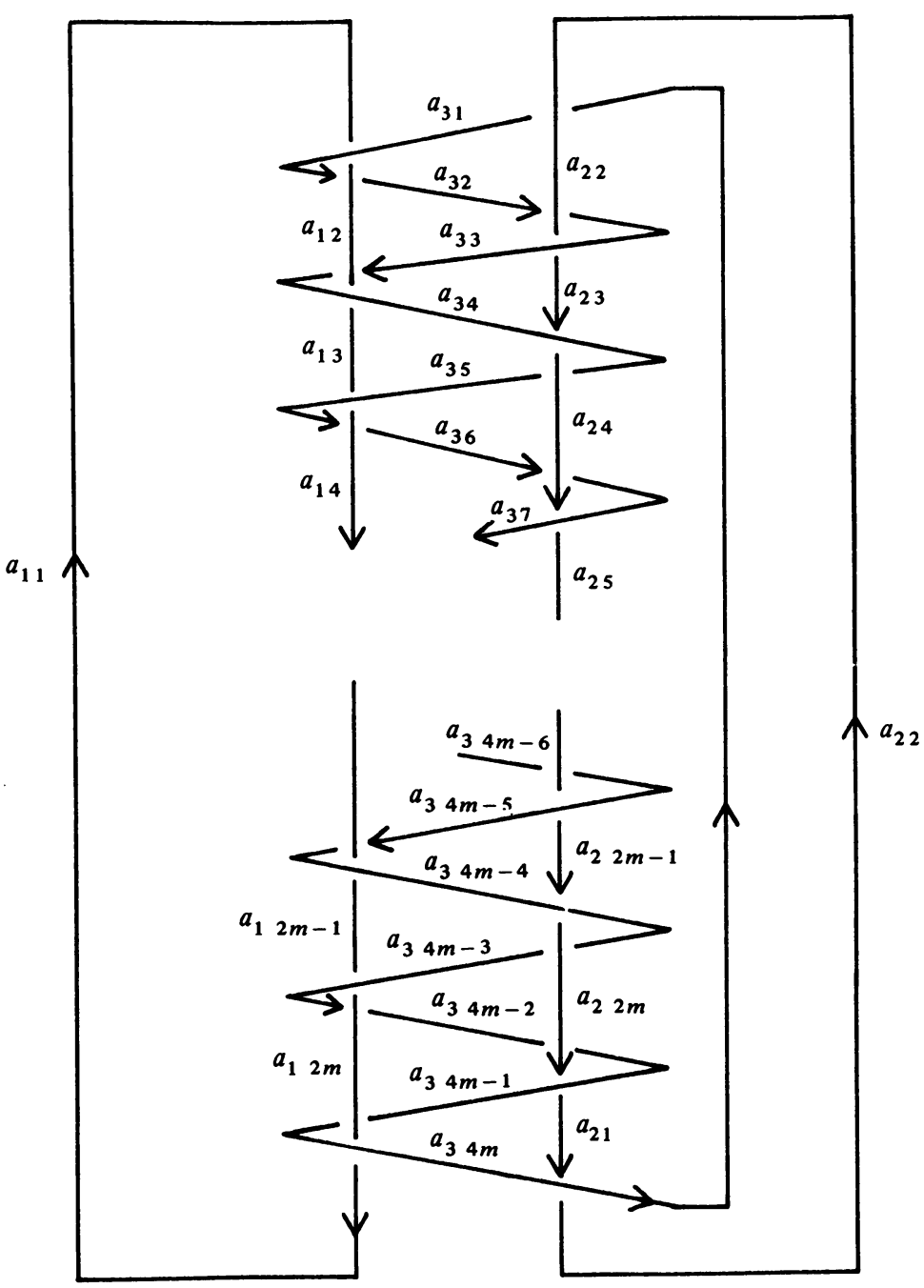

Finally, we point out how some of the Milnor invariants show up in computing the $E_{-s, s}^{s}$ terms. Here then is a brief account of Milnor's work.

In [7] Milnor showed that the group $G / G_{s+1}$, for any nonnegative integer $s$, may be presented by $\left\langle\alpha_{1}, \ldots, \alpha_{n}:\left[\alpha_{i}, \omega_{i}\right], F_{s+1}\right\rangle(i=1, \ldots, n)$, where $\alpha_{i}=a_{i 1}=a_{i}$ represents an ith meridian of $L, \omega_{i}$ is a word in $\alpha_{1}, \ldots, \alpha_{n}$ that represents an ith longitude of $L$ in $G / G_{s+1}$ and $F$ is the free group on $\left\{\alpha_{i}: i=1, \ldots, n\right\}$. 
The Magnus expansion of $\omega_{i}$ is obtained by substituting $\alpha_{j}=1+X_{j}, \alpha_{j}^{-1}=1-$ $X_{j}+X_{j}^{2}-X_{j}^{3}+\cdots$ in the word $\dot{\omega_{i}}$. Thus $\omega_{i}$ can be expressed as a formal, noncommutative power series in the indeterminants $X_{1}, \ldots, X_{n}$. Namely,

$$
\begin{aligned}
\omega_{i}= & +\sum_{j_{1}=1, \ldots, n} \mu\left(j_{1}, i\right) X_{j_{1}}+\sum_{j_{1}, j_{2}=1, \ldots, n} \mu\left(j_{1}, j_{2}, i\right) X_{j_{1}} X_{j_{2}}+\cdots \\
& +\sum_{j_{1} j_{2}, \ldots, j_{t}=1, \ldots, n} \mu\left(j_{1}, j_{2}, \ldots, j_{t}, i\right) X_{j_{1}} X_{j_{2}} \cdots X_{j_{t}}+\cdots
\end{aligned}
$$

Thus a coefficient is defined for each sequence $j_{1}, j_{2}, \ldots, j_{t}, i(t>1)$ of integers between 1 and $n$.

Let $\bar{\Delta}\left(i_{1}, \ldots, i_{r}\right)=$ g.c.d. $\mu\left(j_{1}, \ldots, j_{t}\right)$, where $j_{1}, \ldots, j_{t}(2<t<r-1)$ is to range over all sequences obtained by cancelling at least one of the indices $i_{1}, \ldots, i_{r}$ and permuting the remaining indices cyclically. Then Milnor proved that: the residue classes

$$
\bar{\mu}\left(j_{1}, \ldots, j_{t}, k\right) \equiv \mu\left(j_{1}, \ldots, j_{t}, k\right) \bmod \bar{\Delta}\left(j_{1}, \ldots, j_{t}, k\right)
$$

are isotopy invariants of $L$ provided that $t \leqslant s$.

If we restrict ourselves to links whose $\omega_{i}$ 's belong to $F_{s-1}$ for $(i=1, \ldots, n)$, then $\mu\left(j_{1}, \ldots, j_{t}, i\right)=0$ for $1 \leqslant t \leqslant s-2$. But then $\bar{\mu}\left(j_{1}, \ldots, j_{s-1}, i\right)=$ $\mu\left(j_{1}, \ldots, j_{s-1}, i\right)$, and hence $\mu\left(j_{1}, \ldots, j_{s-1}, i\right)$ are isotopy invariants for such links.

Let $I \bar{F}$ be the kernel of $Z \bar{F} \rightarrow Z$. Let $\bar{N}$ be the ideal of $Z \bar{F}$ generated by $\left[\alpha_{i}, \omega_{i}\right]-1(i=1, \ldots, n)$, and $\bar{F}_{s+1}-1$. Let $\bar{E}$ be the spectral sequence associated with the presentation given by Milnor for the group $G / G_{s+1}$. Now

$$
\bar{E}_{-s, s}^{s}=I^{s} \bar{F} /\left(\bar{N} \cap I^{s} \bar{F}+I^{s+1} \bar{F}\right) \text {. }
$$

If $\omega_{i} \in \bar{F}_{s-1}$, then $\left[\alpha_{i}, \omega_{i}\right]-1 \in I^{s} \bar{F}(i=1, \ldots, n)$ and $\bar{N} \cap I^{s} \bar{F}=\bar{N}$. Hence for this case,

$$
\bar{E}_{-s, s}^{s}=I^{s} \bar{F} /\left(\bar{N}+I^{s+1} \bar{F}\right) \simeq \frac{I^{s} \bar{F} / I^{s+1} \bar{F}}{\left(\bar{N}+I^{s+1} \bar{F}\right) / I^{s+1} \bar{F}} .
$$

Where $I^{s} \bar{F} / I^{s+1} \bar{F}$ is a free $Z$-module write

$$
\left\{X_{i_{1}} X_{i_{2}} \cdots X_{i_{s}}+I^{s+1} \bar{F}: i_{1}, \ldots, i_{s}=1, \ldots, n\right\}
$$

as a basis, and where $\left(\bar{N}+I^{s+1} \bar{F}\right) / I^{s+1} \bar{F}$ is a free $Z$-module generated by $\left\{\left[\alpha_{i}, \omega_{i}\right]-1+I^{s+1} \bar{F}: i=1, \ldots, n\right\}$. But,

$$
\left[\alpha_{i}, \omega_{i}\right]-1=\sum_{j_{1}, \ldots, j_{s-1}=1, \ldots, n}\left[X_{i}, \mu\left(j_{1}, \ldots, j_{s-1}, i\right) X_{j_{1}} X_{j_{2}} \cdots X_{j_{s-1}}\right]+I^{s+1} \bar{F}
$$

Therefore we can replace the set of generators above of the $Z$-module $\left(\bar{N}+I^{s+1} \bar{F}\right) / I^{s+1} \bar{F}$ by the set

$$
\left\{\sum_{j_{1}, \ldots, j_{s-1}=1, \ldots, n}\left[X_{i}, \mu\left(j_{1}, \ldots, j_{s-1}, i\right) X_{j_{1}} \cdots X_{j_{s-1}}\right]+I^{s+1} \bar{F}: i=1, \ldots, n\right\} .
$$

We already proved $E_{-s, s}^{s} \simeq \bar{E}_{-s, s}^{s}$ (see, Theorem (2.11)). We shall describe a precise isomorphism for the case at hand (see, Theorem (3.5)). 


$$
\begin{aligned}
E_{-s, s}^{s} & =\frac{I^{s} F /\left(N_{1}(s)+I^{s+1} F\right)}{\left(N_{2}+N_{1}(s)+I^{s+1} F\right) /\left(N_{1}(s)+I^{s+1} F\right)} \\
& \rightarrow \frac{I^{s} \bar{F} / I^{s+1} \bar{F}}{\left(\bar{N}+I^{s+1} \bar{F}\right) / I^{s+1} \bar{F}}=\bar{E}_{-s, s}^{s}
\end{aligned}
$$

is an isomorphism. From the Wirtinger presentation of $G$ we have $r_{i j}=$ $b_{i j} a_{i j} b_{i j}^{-1} a_{i j+1}^{-1}$. Thus $a_{i j+1}=b_{i j} a_{i j} b_{i j}^{-1}=b_{i j} b_{i j-1} \cdots b_{i 1} a_{i 1} b_{i 1}^{-1} \cdots b_{i j-1}^{-1} b_{i j}^{-1}$. Let $z_{i j}=$ $b_{i j} b_{i j-1} \cdots b_{i 1}$.

Define a sequence of homomorphisms $M_{k}: F \rightarrow \bar{F}$ as follows, by induction on $k$ :

$$
M_{1}\left(a_{i j}\right)=a_{i 1}, \quad M_{k+1}\left(a_{i j+1}\right)=M_{k}\left(z_{i j} a_{i 1} z_{i j}^{-1}\right), \quad M_{k+1}\left(a_{i 1}\right)=a_{i 1} .
$$

Then it can be proved by induction on $k$ that

$$
M_{k}\left(a_{i j}\right)=a_{i j} \bmod \left(F_{k} R\right), \quad M_{k}\left(a_{i j}\right)=M_{k+1}\left(a_{i j}\right) \bmod \left(\bar{F}_{k}\right) .
$$

We claim that $\bar{M}_{s+1}: I F \rightarrow I \bar{F}$, where $\bar{M}_{s+1}$ is the map induced from $M_{s+1}: F \rightarrow \bar{F}$, induces the required isomorphism. Because

$$
M_{s+1}\left(a_{i j}\right)=M_{s+1}\left(z_{i j-1} a_{i 1} z_{i j-1}^{-1}\right)=M_{s+1}\left(z_{i j-1}\right) a_{i 1} M_{s+1}\left(z_{i j-1}^{-1}\right) \equiv a_{i 1} \bmod F_{2} ;
$$

it follows that $\bar{M}_{s+1}\left(u_{i j}-a_{i j} a_{i}^{-1}\right) \in I^{2} \bar{F}$. Hence $M_{s+1}\left(N_{1}(s)\right) \subset I^{s+1} \bar{F}$, moreover, because of $M_{s+1}\left(w_{i k_{i}}\right)=M_{s+1}\left(z_{i k_{i}}\right)=w_{i k_{i}} \bmod \left(F_{s+1} R\right)$. Since $w_{i k_{i}}$ represents an $i$ th longitude of $G, M_{s+1}\left(w_{i k_{i}}\right)$ represents an $i$ th longitude in $G / G_{s+1}$. Let $M_{s+1}$ : $\left(I^{s} F\right) /\left(N_{1}(s)+I^{s+1} F\right) \rightarrow I^{s} \bar{F} / I^{s+1} \bar{F}$ be the canonical homomorphism induced from $\bar{M}_{s+1}$. Then $M_{s+1}$ is an isomorphism, since $I^{s} F /\left(N_{1}(s)+I^{s+1} F\right)$ and $I^{s} \bar{F} / I^{s+1} \bar{F}$ both have rank $n^{s}$. Also,

$$
\bar{M}_{s+1}:\left(N_{2}+N_{1}(s)+I^{s+1} F\right) /\left(N_{1}(s)+I^{s+1} F\right) \rightarrow\left(\bar{N}+I^{s+1} \bar{F}\right) / I^{s+1} \bar{F}
$$

is an isomorphism.

So if one can extract a basis from the generating set (21) of the free $Z$-module $\left(\bar{N}+I^{s+1} \bar{F}\right) / I^{s+1} \bar{F}$, one can then express $E_{-s, s}^{s} \simeq \bar{E}_{-s, s}^{s}$ as a direct sum of a finite number of infinite cyclic groups and cyclic groups of finite order; hence obtaining an explicit demonstration of how the $\mu$ 's appear in $E_{-s, s}^{s}$. For example, for the link described in the figure we have

$$
E_{-s, s}^{s} \simeq \bar{E}_{-s, s}^{s} \simeq Z \oplus \cdots \oplus Z \oplus Z_{m} \oplus Z_{m},
$$

where $m=\mu(1,2,3)=\mu(3,2,1)=\mu(2,3,1)$.

Here are some properties of the Milnor invariants that we will need (see [7]).

(A) The $\bar{\mu}$ satisfy a cyclic symmetry, that is, $\bar{\mu}\left(i_{1}, i_{2}, \ldots, i_{s}\right)=$ $\bar{\mu}\left(i_{\sigma(1)}, i_{\sigma(2)}, \ldots, i_{\sigma(s)}\right)$, where $\sigma$ is a cyclic permutation of $1,2, \ldots, s$. By an invariant $\bar{\mu}\left(i_{1}, \ldots, i_{r+s}\right)$ of type $[r, s]$ will be meant one which involves the index ' 1 ' $r$-times and the index ' 2 ' $s$-times. Then

(B) (i) All invariants of type $[r, 0]$ and $[r, 1](r>2)$ are zero. The invariants of type $[1,1]$ are the linking numbers and these are not necessarily zero.

(ii) All invariants of type $[2 m+1,2]$ are also zero. 
(iii) For the invariants of type $[2 m, 2]$ we have

$$
\begin{aligned}
\bar{\mu}(1, \ldots, 1,2,1,2) & =-\left(\begin{array}{c}
2 m \\
1
\end{array}\right) \bar{\mu}(1, \ldots, 1,1,2,2), \\
\bar{\mu}(1, \ldots, 1,2,1,1,2) & =\left(\begin{array}{c}
2 m \\
2
\end{array}\right) \bar{\mu}(1, \ldots, 1,1,2,2), \\
\bar{\mu}(1, \ldots, 1,2,1,1,1,2) & =-\left(\begin{array}{c}
2 m \\
3
\end{array}\right) \bar{\mu}(1, \ldots, 1,1,2,2), \quad \text { etc. }
\end{aligned}
$$

In view of cyclic symmetry (see (A)) this means that all of the invariants of type $[2 m, 2]$ are completely determined by $\bar{\mu}(1, \ldots, 1,1,2,2)$.

Let $L$ be a two-link. Then

$$
E_{-2,2}^{2} \simeq \frac{I^{2} \bar{F} / I^{3} \bar{F}}{\left(\bar{N}+I^{2} \bar{F}\right) / I^{3} \bar{F}} .
$$

The set $\left\{X_{1}^{2}+I^{3} \bar{F}, X_{2}^{2}+I^{3} \bar{F}, X_{1} X_{2}+I^{3} \bar{F}, X_{2} X_{1}+I^{3} \bar{F}\right\}$ is a basis for $I^{2} \bar{F} / I^{3} \bar{F}$; while $\left(\bar{N}+I^{2} \bar{F}\right) / I^{3} \bar{F}$ is generated by

$\left[\alpha_{1}, \omega_{1}\right]-1=\left[X_{1}, \mu(2,1) X_{2}\right]+I^{3} \bar{F}_{1}, \quad\left[\alpha_{2}, \omega_{2}\right]-1=\left[X_{2}, \mu(1,2) X_{1}\right]+I^{3} \bar{F}$. But $\left[X_{1}, \mu(2,1) X_{2}\right]=-\left[X_{2}, \mu(1,2) X_{1}\right]$. Therefore $\left(\bar{N}+I^{2} \bar{F}\right) / I^{3} \bar{F}$ is a free $Z$ module with basis $\left\{\mu(1,2)\left(X_{1} X_{2}-X_{2} X_{1}\right)+I^{3} \bar{F}\right\}$. But the set $\left\{X_{1}^{2}+I^{3} F, X_{2}^{2}+\right.$ $\left.I^{3} F, X_{1} X_{2}+I^{3} F, X_{1} X_{2}-X_{2} X_{1}+I^{3} \bar{F}\right\}$ may be taken as a basis for $I^{2} \bar{F} / I^{3} \bar{F}$; it follows that

$$
\bar{E}_{-2,2}^{2} \simeq E_{-2,2}^{2} \simeq Z \oplus Z \oplus Z \oplus Z_{\mu(1,2)}
$$

Next assume $\left[\alpha_{i}, \omega_{i}\right]-1 \in I^{3} \bar{F}(i=1,2)$. Then $\left(\bar{N}+I^{3} \bar{F}\right) / I^{4} \bar{F}$ generated by

$$
\begin{aligned}
& {\left[\alpha_{1}, \omega_{1}\right]-1=\sum_{j_{1}, j_{2}=1,2}\left[X_{1}, \mu\left(j_{1}, j_{2}, 1\right) X_{j_{1}} X_{j_{2}}\right]+I^{4} \bar{F},} \\
& {\left[\alpha_{2}, \omega_{2}\right]-1=\sum_{j_{1}, j_{2}=1,2}\left[X_{2}, \mu\left(J_{1}, j_{2}, 2\right) X_{j_{1}} X_{j_{2}}\right]+I^{4} \bar{F} .}
\end{aligned}
$$

But, all the $\mu\left(j_{1}, j_{2}, i\right), i=1,2$, appearing above are zero, due to properties (B) (i) and (B) (ii). Hence nothing could be said about such a link by looking at $\bar{E}_{-3,3}^{3}$. So we consider the case $\left[\alpha_{i}, \omega_{i}\right]-1 \in I^{4} \bar{F}(i=1,2)$. Then $\left(\bar{N}+I^{4} \bar{F}\right) / I^{5} \bar{F}$ is generated by

$$
\begin{aligned}
& {\left[\alpha_{1}, \omega_{1}\right]-1=\mu(1,1,2,2)\left(X_{1}^{2} X_{2}^{2}+2 X_{2} X_{1} X_{2} X_{1}-2 X_{1} X_{2} X_{1} X_{2}-X_{2}^{2} X_{1}^{2}\right)+I^{5} \bar{F},} \\
& {\left[\alpha_{2}, \omega_{2}\right]-1=\mu(1,1,2,2)\left(X_{2}^{2} X_{1}^{2}+2 X_{1} X_{2} X_{1} X_{2}-2 X_{2} X_{1} X_{2} X_{1}-X_{1}^{2} X_{2}^{2}\right)+I^{5} \bar{F} .}
\end{aligned}
$$

Hence $\left(\bar{N}+I^{4} \bar{F}\right) / I^{5} \bar{F}$ is a free $Z$-module with basis the vector

$$
\mu(1,1,2,2)\left(X_{2}^{2} X_{1}^{2}+2 X_{1} X_{2} X_{1} X_{2}-2 X_{2} X_{1} X_{2} X_{1}-X_{1}^{2} X_{2}^{2}\right)+I^{5} \bar{F} .
$$

The free $Z$-module $I^{4} \bar{F} / I^{5} \bar{F}$ has rank 16 . Hence the spectral sequence term

$$
E_{-4,4}^{4} \simeq E_{-4,4}^{4} \simeq Z \oplus \cdots \oplus Z \oplus Z_{\mu(1,1,2,2)},
$$

where there are fifteen copies of $Z$ in the summand. 
Thus, for the special links whose longitudes belong to $I^{s} F$ the term $E_{-s, s}^{s}$ sheds light on the Milnor invariants. Naturally one would like to do this study for more general links. The calculations are similar to those in [8].

\section{REFERENCES}

1. R. Baer, The higher commutator subgroups of a group, Bull. Amer. Math. Soc. 50 (1944), 143-160.

2. K. T. Chen, Isotopy invariants of links, Ann. of Math. (2) 56 (1952), 343-353.

3. R. H. Fox, Free differential calculus. I, Ann. of Math. (2) 57 (1953), 547-560.

4. A. Frohlich, Baer invariants of algebras, Trans. Amer. Math. Soc. 109 (1963), 221-244.

5. M. Gutiérrez, The $\bar{\mu}$-invariants for groups, Proc. Amer. Math. Soc. 55 (1976), 293-298.

6. P. Hilton and U. Stammbach, $A$ course in homological algebra, Springer-Verlag, New York, 1971.

7. J. Milnor, Isotopy of links, Lefschetz Symposium, Princeton Univ. Press, Princeton, N. J., 1957.

8. N. Smythe, Isotopy tmoariants for links and the Alexander matrix, Amer. J. Math. 89 (1967), 693-703.

9. J. Stallings, Quotients of the powers of the augmentation ideal in a gnoup ring, Ann. of Math. Studies No. 84, Princeton Univ. Press, Princeton, N. J., 1975, pp. 101-118.

Department of Mathematics, Bowling Green State University, Bowlnng Green, Oho 43403 